\title{
SPECTRAL TYPE OF THE SHIFT TRANSFORMATION OF DIFFERENTIAL PROCESSES WITH STATIONARY INCREMENTS $\left({ }^{1}\right)$
}

\author{
BY \\ KIYOSI ITÔ
}

1. Introduction. Before discussing our problems on stochastic processes, we shall define two kinds of equivalence of groups of measure preserving set transformations following J. v. Neumann and P. R. Halmos [8]( $\left.{ }^{2}\right)$. By a measure preserving set transformation we understand a mapping from the system of measurable sets of one measure space onto that of another measure space modulo null sets which preserves measure and set operations such as countable sum and complement. Given a measure space $\Omega(B, m)$ and a oneparameter group $\left\{T_{\tau}\right\}$ of measure preserving set transformations from $\boldsymbol{B}$ onto itself, let $H$ denote the $L^{2}$-space over $\Omega$. The above group $\left\{T_{\tau}\right\}$ will induce a group $\left\{U_{\tau}\right\}$ of unitary operators on $H$ such that

$$
U_{\tau} \chi_{M}=\chi_{M[\tau]}
$$

where $M$ is any set of finite $m$-measure, $M[\tau]=T_{\tau} M$, and $\chi_{A}$ denotes the characteristic function of a set $A$. We consider another measure space $\bar{\Omega}(\bar{B}, \bar{m})$ associated with a group of measure preserving set transformations $\left\{\bar{T}_{\tau}\right\}$ and we define $\bar{H}$ and $\left\{\bar{U}_{\tau}\right\}$ correspondingly. If there exists a measure preserving set transformation $S$ from $B$ onto $\bar{B}$ such that

$$
\bar{T}_{\tau}=S T_{\tau} S^{-1},
$$

then we say that $\left\{\bar{T}_{\tau}\right\}$ and $\left\{T_{\tau}\right\}$ are of the same spatial type. We shall also introduce another classification of transformation groups which is rougher than the above. If there exists an isometric linear mapping $V$ from $\boldsymbol{H}$ onto $\overline{\boldsymbol{H}}$ such that

$$
\bar{U}_{\tau}=V U_{\tau} V^{-1},
$$

then we say that $\left\{T_{\tau}\right\}$ and $\left\{\bar{T}_{\tau}\right\}$ are of the same spectral type.

Let $X(t, \omega),-\infty<t<\infty$, be a measurable (in two variables $t$ and $\omega$ ) differential process with stationary increments on a probability space $\Omega(B, P)$. For any finite interval $I=(s, t]$ we shall define the increment $\Delta X(I, \omega)$ as $X(t, \omega)-X(s, \omega)$. By $B_{X}$ we denote the Borel system of subsets of $\Omega$ generated

Received by the editors May 25, 1955.

(1) This work was supported by a research project at Princeton University sponsored by the Office of Ordnance Research, DA-36-034-ORD-1296 RD.

(2) Numbers in brackets refer to the references at the end of this paper. 
by all the sets of the form $\{\omega ; \Delta X(I, \omega)<c\}$, and we shall consider the measure space $\Omega\left(B_{X}, P\right)$ and the $L^{2}$-space over it, say $H_{X}$. Now we shall define a one-parameter group $\left\{T_{\tau}\right\}$ of measure preserving set transformations (shift transformation) on $B_{X}$ by

$$
T_{\tau}\{\omega ; \Delta X(I, \omega)<c\}=\{\omega ; \Delta X(I+\tau, \omega)<c\},
$$

where $I+\tau$ is the interval $\{x+\tau ; x \in I\}$. The possibility of this definition follows from the definition of differential processes with stationary increments. From $\left\{T_{\tau}\right\}$ we can derive a group $\left\{U_{\tau}\right\}$ of unitary operators on $H_{X}$ as above.

The purpose of this paper is to determine the spectral type of this group $\left\{T_{\tau}\right\}$. In $\S 2$ we shall summarize some known facts on differential processes as preliminaries. In $\S 3$ and $\S 4$ we shall introduce a multiple Wiener integral which will play an important role in our theory. Our aim will be attained in $\S 5$. The fundamental theorem established there generalizes Kakutani's theorem [6] on the spectra of the flow of Brownian motion and implies that the transformation groups induced by different processes are of the same spectral type. But it is still an open question whether they are of the same spatial type or not.

2. Independent random measure associated with a measurable differential process with stationary increments. Let $\Xi$ be a measurable space on which a class of sets called measurable, $B_{\Xi}^{\prime}$, is assigned so that (1) the empty set $\varnothing \in B_{\Xi}^{\prime}$, (2) $E_{1}, E_{2} \in B_{\Xi}^{\prime}$ implies $E_{1} \cup E_{2}, E_{1}-E_{2} \in B_{\Xi}^{\prime}$, and (3) any decreasing sequence in $B_{\Xi}^{\prime}$ has its limit set in $\boldsymbol{B}_{\Xi}^{\prime}$; we do not assume that the whole space $\Xi$ belongs to $B_{\Xi}^{\prime}$.

A system of random variables $f(E)=f(E, \omega)$ depending on a set $E \in B_{z}^{\prime}$ with a probability parameter $\omega$ is called an independent random measure if $f\left(E_{1}\right), f\left(E_{2}\right), \cdots, f\left(E_{n}\right)$ are independent and

$$
f\left(\bigcup_{i=1}^{n} E_{i}\right)=\sum_{i=1}^{n} f\left(E_{i}\right)
$$

for every finite system $\left\{E_{i}\right\}$ of disjoint sets in $B_{\Xi}$ and if

$$
f\left(E_{n}\right) \rightarrow 0 \text { (convergence in probability) }
$$

for every decreasing sequence $\left\{E_{n}\right\}$ in $B_{\Xi}^{\prime}$ tending to the empty set. We can easily show that the above conditions imply that (2.1.b) holds in the sense of almost everywhere convergence. A normal random measure [4] is a special case of an independent random measure.

Let $X(t, \omega)$ be a measurable differential process with stationary increments. By taking Doob's separable modification [1] of this process, we may assume that $X(t, \omega)$ is continuous in $t$ except for discontinuities of the first kind with probability 1 . We also assume, as we may, that $X(\tau, \omega)$ is continuous on the right in $\tau$ with probability 1 . By a theorem of P. Lévy [7], $\Delta X(I, \omega)$ 
is subject to an infinitely divisible law whose characteristic function $\phi_{I}(z)$ is given by

$$
\log \phi_{I}(z)=|I|\left[i \gamma z+\int_{-\infty}^{\infty}\left(e^{i z u}-1-\frac{i z u}{1+u^{2}}\right) \frac{1+u^{2}}{u^{2}} d \beta(u)\right]
$$

where $|I|$ means the length of $I, \gamma$ is a real constant, and $d \beta$ is a bounded measure on $(-\infty, \infty)$.

We shall consider a plane $\pi$ on which a coordinate system $(t, u)$ is assigned. Let $B_{\pi}$ denote the class of all Borel subsets of $\pi$. We shall consider two measures $\nu, \mu$ on $\pi\left(B_{x}\right)$ by

$$
\begin{aligned}
& \nu(E)=\iint_{E} \frac{1+u^{2}}{u^{2}} d t d \beta(u), \\
& \mu(E)=\iint_{E}\left(1+u^{2}\right) d t d \beta(u)=\iint_{E} u^{2} d \nu(t, u) .
\end{aligned}
$$

It is clear that $\mu$ is the product of the one-dimensional measures $d t$ and $d \mu^{\prime}(u)=\left(1+u^{2}\right) d \beta(u)$. Let $\widetilde{B}_{\pi}$ be the totality of bounded Borel subsets of $\pi$ whose distance from the $t$-axis is positive. We may consider $\pi\left(\widetilde{B}_{\pi}\right)$ as a measurable space. If we define $N(E, \omega), E \in \widetilde{B}_{\pi}$, to be the number of points $(t, u) \in E$ for which $X(t, \omega)-X(t-0, \omega)=u$. Then $N(E, \omega)$ is subject to Poisson distribution with mean $\nu(E)$ for $E$ fixed and the system $\{N(E, \omega)$, $\left.E \in \widetilde{B}_{\pi}\right\}$ is an independent random measure. Further we have the following expression of $X(t, \omega)$ :

$$
\begin{aligned}
\Delta X(I, \omega)= & \gamma|I|+\sigma \Delta B(I, \omega) \\
& +\lim _{n \rightarrow \infty} \int_{t \in I} \int_{n^{-1}<|u|<n}\left[u d N(t, u)-\frac{u}{1+u^{2}} d \nu(t, u)\right]
\end{aligned}
$$

where $\sigma^{2}=\beta(+0)-\beta(-0)$ and $B(t, \omega)$ is a Wiener process which is independent of the system $\left\{N(E, \omega) ; E \in \widetilde{B}_{\pi}\right\}$. We can easily deduce these facts from the results stated in [3].

Next we shall introduce another independent random measure $M(E)$. Let $B_{\pi}^{*}$ be the class of all Borel subsets of $\pi$ whose $\mu$-measure is finite. For $E$ in $B_{\pi}^{*}$ we define $M(E)$ by

$$
M(E)=M(E, \omega)=\int_{E(0)} \sigma \cdot d B(t)+\lim _{n \rightarrow \infty} \iint_{(t, u) \in E(n)}[u d N(t, u)-u d v(t, u)],
$$

where

$$
\begin{aligned}
& E(n)=\left\{(t, u) \in E ; n^{-1}<|u|<n\right\}, \quad n=1,2, \cdots, \\
& E(0)=\{t ;(t, 0) \in E\}
\end{aligned}
$$


and the integral based on $d B(t)$ is the Wiener integral [1, IX, 2].

It is to be noted that $N(E), \Delta B(I)$ and $M(E)$ belong to $H_{X}$ for $E$ and $I$ fixed. Also the expectation of $M(E)$ is 0 .

3. Definition of multiple Wiener integral based on $d M$. Let $M(E, \omega)$, $E \in B_{\pi}^{*}$, be the independent random measure defined in $\S 2$. We can easily verify the following properties:

$$
\begin{aligned}
\left(M\left(E_{1}\right), M\left(E_{2}\right)\right) & =\mu\left(E_{1} \cap E_{2}\right) \\
\|M(E)\|^{2} & =\mu(E), \\
(M(E), 1) & =0 .
\end{aligned}
$$

In this section we shall make use of the following property of $\mu$ :

Continuity: For every $E \in B_{\pi}^{*}$ and every $\epsilon>0$, there exists a finite subdivision of $E: E=E_{1} \cup E_{2} \cup \ldots \cup E_{n}$ such that $\mu\left(E_{i}\right)<\epsilon, 1 \leqq i \leqq n$.

Let $\pi^{p}$ be the product measure space $\left[\pi\left(B_{\pi}, \mu\right)\right]^{p}$ and let $L_{p}^{2}$ be the $L^{2}$-space over $\pi^{p}$. We shall denote points of $\pi$ by $\xi=(t, u), \xi^{\prime}=\left(t^{\prime}, u^{\prime}\right), \xi_{i}=\left(t_{i}, u_{i}\right)$, etc. For any $f \in L_{p}^{2}$ we shall define the symmetrized function $\tilde{f}$ of $f$ to be

$$
\tilde{f}\left(\xi_{1}, \cdots, \xi_{p}\right)=\frac{1}{p !} \sum_{(\epsilon)} f\left(\xi_{\epsilon}(1), \cdots, \xi_{\epsilon}(p)\right)
$$

where $(\epsilon)=(\epsilon(1), \cdots, \epsilon(p))$ runs over all arrangements of $(1,2, \cdots, p)$. It is clear that $f\left(\xi_{1}, \cdots, \xi_{p}\right)$ is symmetric in $\left(\xi_{1}, \cdots, \xi_{p}\right)$ if and only if it coincides with $\bar{f} . \bar{f}$ is symmetric. We have also

$$
\|\tilde{f}\| \leqq\|f\|, \quad\|\| \text { being the norm in } L_{p}^{2} .
$$

Let $C_{p}$ denote the class of all functions of the form:

$$
c_{p}\left(\xi_{1}, \xi_{2}, \cdots, \xi_{p}\right)=\chi\left(\xi_{1}, E_{1}\right) \chi\left(\xi_{2}, E_{2}\right) \cdots \chi\left(\xi_{p}, E_{p}\right)
$$

where $E_{i}, i=1,2, \cdots, p$, are disjoint sets in $B_{\pi}^{*}$ and $\chi(\xi, E)$ denotes the characteristic function of the set $E$. Let $S_{p}$ denote the class of all linear combinations of functions in $C_{p}$. The continuity of $\mu$ implies that $S_{p}$ is dense in $L_{p}^{2}$ [4, Theorem 2.1].

Now we shall define the multiple Wiener integral of the pth degree based on the independent random measure $M$ and denote it by

$$
I_{p}(f)=\int \cdots \int f\left(\xi_{1}, \cdots, \xi_{p}\right) d M\left(\xi_{1}\right) \cdots d M\left(\xi_{p}\right) .
$$

\section{We define}

$$
I_{p}\left(c_{p}\right)=M\left(E_{1}\right) \cdots M\left(E_{p}\right)
$$

for the $c_{p}$ of (3.5), then $I_{p}(s)$ for $s$ in $S_{p}$ by linearity and finally $I_{p}(f)$ for $f$ in $L_{p}^{2}$ by continuity. For convenience of notation we shall denote by $L_{0}^{2}$ the com- 
plex number field (one-dimensional Hilbert space) and define $I_{0}(c)=c$ if $c \in L_{0}^{2}$. In exactly the same way as in the case of normal random measure [4] we can show that the definition is possible and that the following theorem holds.

THEOREM 1.

$$
\begin{aligned}
I_{p}(f) & =I_{p}(\tilde{f}), \\
I_{p}(a f+b g) & =a I_{p}(f)+b I_{p}(g), \\
\left(I_{p}(f), I_{p}(g)\right) & =p ! \cdot(\tilde{f}, \tilde{g}), \\
\left\|I_{p}(f)\right\| & =(p !)^{1 / 2}\|\tilde{f}\| \leqq(p !)^{1 / 2}\|f\|, \\
\left(I_{p}(f), I_{q}(g)\right) & =0
\end{aligned}
$$$$
(p \neq q) .
$$

4. Completeness of multiple Wiener integrals. Let $\tilde{L}_{p}^{2}$ denote the class of all symmetric functions in $L_{p}^{2}$. $\tilde{L}_{p}^{2}$ is clearly a closed linear manifold of $L_{p}^{2}$. By (I.1) in Theorem 1 we see that the image of $\tilde{L}_{p}^{2}$ by $I_{p}$ coincides with that of $L_{p}^{2}$, which we shall denote by $\boldsymbol{H}_{X}^{(p)}$. Since the mapping:

$$
V_{p}: \tilde{L}_{p}^{2} \ni f_{p} \rightarrow(p !)^{-1 / 2} I_{p}\left(\tilde{f}_{p}\right) \in \boldsymbol{H}_{\boldsymbol{X}}
$$

is isometric by $\left(\mathrm{I} .3^{\prime}\right), \boldsymbol{H}_{X}^{(p)}$ is a closed linear manifold of $\boldsymbol{H}_{X}$ isomorphic with $\tilde{L}_{p}^{2}$ by $V_{p}$. By (I.4) we see that the $\boldsymbol{H}_{X}^{(p)}, p=0,1,2, \cdots$, are orthogonal to each other.

We shall establish the following theorem which implies the completeness of $\left\{I_{p}\left(\tilde{f}_{p}\right) ; \tilde{f}_{p} \in \tilde{L}_{p}^{2}, p=0,1,2, \cdots\right\}$.

THEOREM 2.

$$
H_{X}=\sum_{p \geqq 0} \oplus H_{X}^{(p)} \quad\left(\sum \oplus\right. \text { means 'direct sum'). }
$$

Proof. $1^{\circ}$. We shall first prove the following lemma.

LEMma 1. All the elements of the following form constitute a fundamental set in $H_{X}$ :

$$
N\left(E_{1}\right)^{p_{1}} \cdots N\left(E_{m}\right)^{p_{m}} \Delta B\left(I_{1}\right)^{q_{1}} \cdots \Delta B\left(I_{n}\right)^{q_{n}},
$$

where $m, n=1,2, \cdots, p_{i}, q_{j}=0,1,2, \cdots$, and $\left\{E_{j}\right\}$ and $\left\{I_{j}\right\}$ are each disjoint.

Proof $\left({ }^{3}\right)$. It is clear that an element of the form (4.2) belongs to $H_{X}$, since it has a finite norm. By the definition of $\boldsymbol{H}_{X}$ we can easily see that the totality of the elements of $\boldsymbol{H}_{X}$ of the following form constitute a fundamental set in $H_{X}$ :

${ }^{3}$ The author owes this proof to I. E. Segal who has established a more general fact in his paper [9]. 


$$
Y=f\left(\Delta X\left(I_{1}\right), \cdots, \Delta X\left(I_{n}\right)\right),
$$

where $n=1,2, \cdots,\left\{I_{i}\right\}$ are disjoint and $f$ is a bounded continuous function. By the expression of (2.5), $\Delta X(I)$ is the limit of linear combinations of $\{N(E)\}$ and $\{\Delta B(I)\}$. Therefore the elements of the following form also constitute a fundamental set in $\boldsymbol{H}_{X}$ :

$$
Z=f\left(N\left(E_{1}\right), \cdots, N\left(E_{m}\right), \quad \Delta B\left(I_{1}\right), \cdots, \Delta B\left(I_{n}\right)\right)
$$

where the $E_{i}$ are pairwise disjoint, the $I_{j}$ are also pairwise disjoint and $f$ is a bounded continuous function.

Let $\mathfrak{M}=\mathfrak{M}\left(E_{1}, \cdots, E_{m}, I_{1}, \cdots, I_{n}\right)$ be the closed linear manifold spanned by the polynomials in $N\left(E_{1}\right), \cdots, N\left(E_{m}\right), \Delta B\left(I_{1}\right), \cdots, \Delta B\left(I_{n}\right)$. To prove the lemma it is enough to show that $Z$ in (4.4) belongs to $\mathfrak{M}$. We put

$$
Z=U+V=g\left(N\left(E_{1}\right), \cdots, \Delta B\left(I_{n}\right)\right)+h\left(N\left(E_{1}\right), \cdots, \Delta B\left(I_{n}\right)\right)
$$

where $U \in \mathfrak{M}, V \perp \mathfrak{M}$, and both $g$ and $h$ are Baire functions. It is enough to show $V=0$. To avoid trivial complications we consider the case that $m=n=1$. It is sufficient to derive $h(N(E), \Delta B(I))=0$ for almost all $\omega$ from the following conditions:

$$
\left(h(N(E), \Delta B(I)), \quad N(E)^{p} \Delta B(I)^{q}\right)=0, \quad p, q=0,1,2, \cdots .
$$

We denote by $\sigma_{1}$ and $\sigma_{2}$ respectively the distribution of $N(E)$ (Poisson distribution) and that of $\Delta B(I)$ (normal distribution). Then (4.5) can be written as

$$
\iint h(x, y) x^{p} y^{q} d \sigma_{1}(x) d \sigma_{2}(y)=0
$$

But we have

$$
\begin{aligned}
\iint e^{|\ell x|+|s y|}|h(x, y)| d \sigma_{1}(x) d \sigma_{2}(y) \\
\quad \leqq \\
\quad=\|h(N(E), B(I))\|\left(\int e^{2|t x|} d \sigma_{1}(x) \int e^{2|s y|} d \sigma_{2}(y)\right)^{1 / 2} \\
<\infty .
\end{aligned}
$$

Therefore $\left(4.5^{\prime}\right)$ will imply

$$
\iint h(x, y) e^{i(t x+s y)} d \sigma_{1}(x) d \sigma_{2}(y)=0, \quad-\infty<t, s<\infty .
$$


Thus we have $h(x, y)=0$ for almost all $(x, y)$ with respect to the measure $d \sigma_{1}(x) d \sigma_{2}(y)$, that is, $h(N(E), \Delta B(I))=0$ for almost all $\omega$.

$2^{\circ}$. Lemma 2. The elements of the following form constitute a fundamental set in $H_{X}$ :

$$
Y=N\left(E_{1}\right) \cdots N\left(E_{m}\right) \Delta B\left(I_{1}\right) \cdots \Delta B\left(I_{n}\right),
$$

where the $E_{i}$ are pairwise disjoint and the $I_{j}$ are also pairwise disjoint.

Proof. Making use of the fact that the independence of $X$ and $Y$ implies $\|X Y\|=\|X\| \cdot\|Y\|$, we can easily see the following fact:

$\left(^{*}\right)$ Let $\left\{X_{1}, X_{2} \cdots\right\}$ and $\left\{Y_{1}, Y_{2} \cdots\right\}$ be two independent sequences of random variables. If $X_{n} \rightarrow X$ and $Y_{n} \rightarrow Y$ in $L^{2}(\Omega)$, then $X_{n} Y_{n} \rightarrow X Y$ in $L^{2}(\Omega)$.

Let $N$ denote the closed linear manifold in $H_{X}$ spanned by the elements of the form $N\left(E_{1}\right) N\left(E_{2}\right) \cdots N\left(E_{m}\right)$ with pairwise disjoint $E_{i}$ and let $B$ be the closed linear manifold spanned by the elements of the form $\Delta B\left(I_{1}\right) \Delta B\left(I_{2}\right)$ $\cdots \Delta B\left(I_{n}\right)$ with pairwise disjoint $I_{j}$. By the above remark $\left(^{*}\right)$ it is enough to show that

$$
N\left(E_{1}\right)^{p_{1}} N\left(E_{2}\right)^{p_{2}} \cdots N\left(E_{m}\right)^{p_{m}} \in \boldsymbol{N}
$$

and

$$
\Delta B\left(I_{1}\right)^{q_{1}} \Delta B\left(I_{2}\right)^{q_{2}} \cdots \Delta B\left(I_{n}\right)^{q_{n}} \in B
$$

whenever the $E_{i}$ or the $I_{j}$ are disjoint. (4.8) was proved in [4, Theorem 4.2].

It remains to prove (4.7). Consider a subdivision $\left\{F_{i}\right\}, i=1, \cdots, s$, of $\left\{E_{i}\right\}, i=1, \cdots, m$, so fine that $\nu\left(F_{i}\right)<\operatorname{Min}(\epsilon / \nu(E), 1)$ where $\epsilon>0$ and $E=F_{1} \cup F_{2} \cup \ldots \cup F_{\text {s. }}$ Then we have the following expression:

$$
N=N\left(E_{1}\right)^{p_{1}} N\left(E_{2}\right)^{p_{2}} \cdots N\left(E_{m}\right)^{p_{m}}=\sum N\left(F_{i(1)}\right)^{i_{1}} \cdots N\left(F_{i(r)}\right)^{i_{r}}
$$

with $i(1)<i(2)<\cdots<i(r)$.

Since $N\left(F_{i}\right)$ takes only non-negative integral values, we have

$$
N \geqq \sum N\left(F_{i(1)}\right) N\left(F_{i(2)}\right) \cdots N\left(F_{i(r)}\right) \equiv N_{\epsilon} \in \boldsymbol{N},
$$

and also

$$
\begin{aligned}
P\left(N \neq N_{\epsilon}\right) & =P\left(N\left(F_{i}\right) \geqq 2 \text { for some } i\right) \\
& \leqq \sum_{i=1}^{s} P\left(N\left(F_{i}\right) \geqq 2\right) \leqq \sum_{i=1}^{s} \nu\left(F_{i}\right)^{2}<\epsilon .
\end{aligned}
$$

Therefore $N_{\epsilon} \rightarrow N$ in probability as $\epsilon \rightarrow 0$. Thus we can choose a sequence $\epsilon(n) \quad(\rightarrow 0)$ such that $N_{\epsilon(n)} \rightarrow N$ almost everywhere in $\omega$. Further we have $0 \leqq N_{\epsilon(n)} \leqq N$ and $N \in L^{2}(\Omega)$. Therefore we have $\left\|N_{\epsilon(n)}-N\right\| \rightarrow 0$ and so $N \in N$.

$3^{\circ}$. Now we shall deduce Theorem 2 from Lemma 2. It is enough to show that $Y$ in (4.6) is expressible as 


$$
Y=I_{0}\left(f_{0}\right)+I_{1}\left(f_{1}\right)+\cdots+I_{m+n}\left(f_{m+n}\right) .
$$

Using the notation in $\S 3$, we set

$$
\begin{aligned}
f\left(\xi_{1}, \cdots, \xi_{m}, \xi_{m+1}\right. & \left.\cdots, \xi_{m+n}\right) \\
& =u_{1}^{-1} \cdots u_{m}^{-1} \sigma^{-n} \chi_{E_{1}}\left(\xi_{1}\right) \cdots \chi_{E_{n}}\left(\xi_{n}\right) \chi_{I_{1}}\left(\xi_{m+1}\right) \cdots \chi_{I_{m+n}}\left(\xi_{m+n}\right),
\end{aligned}
$$

where $\chi_{I}$ denote the characteristic function of the interval $I \times\{0\}$ (in the plane $\pi)$ and $\xi_{i}=\left(t_{i}, u_{i}\right)$. Then we have

$$
\begin{aligned}
I_{m+n}(f) & =\left(N\left(E_{1}\right)-\nu\left(E_{1}\right)\right) \cdots\left(N\left(E_{m}\right)-\nu\left(E_{m}\right)\right) \Delta B\left(I_{1}\right) \cdots \Delta B\left(I_{n}\right) \\
& =N\left(E_{1}\right) \cdots N\left(E_{m}\right) \Delta B\left(I_{1}\right) \cdots \Delta B\left(I_{n}\right)+R,
\end{aligned}
$$

where $R$ is a linear combination of the elements of the form:

$$
N\left(E_{i(1)}\right) \cdots N\left(E_{i(p)}\right) \Delta B\left(I_{1}\right) \cdots \Delta B\left(I_{n}\right), \quad p<m,
$$

and $R=0$ in case $m=0$. Therefore we obtain (4.9) by induction on $m$. Thus our theorem is completely proved.

5. Spectral type of $\left\{T_{\tau}\right\}$. In the last section we proved that

$$
H_{X}=\sum_{p \geqq 0} \oplus H_{X}^{(p)}
$$

and that each $\boldsymbol{H}_{X}^{(p)}$ is isomorphic to $\tilde{L}_{p}^{2}$ by $V_{p}$. Now we shall investigate the behavior of the group of unitary transformations $U_{\tau}$ on $H_{X}$ derived from the shift transformations $T_{\tau}$ of the process $X(t, \omega)$.

Let $U_{\tau}^{(p)}$ be the restriction of $U_{\tau}$ to $H_{X}^{(p)}$. Then we have

LEMMA 1. $U_{\tau}^{(p)}$ is a unitary operator on $H_{X}^{(p)}$ which is transformed by $V_{p}$ into the following unitary operator $\tilde{S}_{\tau}^{(p)}$ on $\tilde{L}_{p}^{2}$.

$$
\tilde{S}_{\tau}^{(p)} \tilde{f}\left(t_{1}, u_{1}, \cdots, t_{p}, u_{p}\right)=\tilde{f}\left(t_{1}-\tau, u_{1}, \cdots t_{p}-\tau, u_{p}\right), \quad \tilde{f} \in \tilde{L}_{p}^{2} .
$$

Corresponding to the decomposition (5.1), we have

$$
U_{\tau}=\sum_{p \geqq 0} \oplus U_{\tau}^{(p)}
$$

Proof. By the same expression as in (5.2) we shall define a unitary operator $S_{\tau}^{(p)}$ on $L_{p}^{2}$. It is clear that $S_{\tau}^{(p)}$ is an extension of $\widetilde{S}_{\tau}^{(p)}$. Then we have

$$
I_{p}\left(S_{\tau}^{(p)} f\right)=U_{\tau} I_{p}(f) .
$$

This is clear by the definition if $f$ has the form (3.5), and so it holds also if $f \in L_{p}^{2}$, because both sides of (5.4) are bounded and linear in $f$.

Since $U_{\tau}$ transforms $\boldsymbol{H}_{X}^{(p)}$ onto $\boldsymbol{H}_{X}^{(p)}$ by (5.4), the restriction $U_{\tau}^{(p)}$ of $U_{\tau}$ to 
$H_{X}^{(p)}$ is a unitary operator on $H_{X}^{(p)}$. By (5.4) and the definition of $V_{p}$ and $\tilde{S}_{r}^{(p)}$ we have

$$
U_{\tau}^{(p)}=V_{p} \tilde{S}_{\tau}^{(p)} V_{p}^{-1}
$$

Thus we can easily verify (5.3), since $U_{\tau}$ is a unitary operator on $H_{X}$.

Lemma 1 reduces the investigation of $\left\{U_{\tau}\right\}$ to that of $\left\{\widetilde{S}_{\tau}^{(p)}\right\} . \widetilde{S}_{\tau}^{0}$ is only the identity operator on $L_{0}^{2}$, which is a one-dimensional Hilbert space. We consider $\tilde{S}_{\tau}^{(p)}, p \geqq 1$. We shall introduce two transformation groups, $\{(\tau)\}$ and $\{(\epsilon)\}$, on the $2 p$-dimensional space $\pi^{p}$ as follows:

$$
\begin{aligned}
& (\tau)\left(t_{1}, u_{1}, t_{2}, u_{2}, \cdots, t_{p}, u_{p}\right)=\left(t_{1}-\tau, u_{1}, t_{2}-\tau, u_{2}, \cdots, t_{p}-\tau, u_{p}\right), \\
& (\epsilon)\left(t_{1}, u_{1}, t_{2}, u_{2}, \cdots, t_{p}, u_{p}\right)=\left(t_{\epsilon(1)}, u_{\epsilon(1)}, t_{\epsilon(2)}, u_{\epsilon(2)}, \cdots, t_{\epsilon(p)}, u_{\epsilon(p)}\right),
\end{aligned}
$$

where $\tau$ is a real number and $\epsilon=\{\epsilon(1), \epsilon(2), \cdots, \epsilon(p)\}$ is an arrangement of $\{1,2, \cdots, p\}$.

To make it easier to see how these transformations act on $\pi^{p}$, we shall consider the following coordinate transformation:

$\left(t_{1}, u_{1}, t_{2}, u_{2}, \cdots, t_{p}, u_{p}\right)$

$$
\rightarrow\left(t, s_{2}, \cdots, s_{p-1}, u_{1}, \cdots, u_{p}\right)=(t, v), v \in R^{2 p-1}
$$

which is defined by

$$
\begin{aligned}
t & =\frac{1}{p}\left(t_{1}+t_{2}+\cdots+t_{p}\right), \\
s_{i} & =\sum_{j=1}^{p} \alpha_{i j} t_{j}, \quad i=1,2, \cdots, p-1,
\end{aligned}
$$

where $\left(\alpha_{i j} ; 1 \leqq i \leqq p-1,1 \leqq j \leqq p\right)$ is a certain real matrix satisfying

$$
\begin{gathered}
\sum_{i} \alpha_{i j}=0, \\
\left|\begin{array}{ccc}
1 / p & \cdots & 1 / p \\
\alpha_{11} & \cdots & \alpha_{1 p} \\
\cdot & \cdots & \cdot \\
\alpha_{p-1,1} & \cdots & \alpha_{p-1, p}
\end{array}\right|=1 .
\end{gathered}
$$

By (5.8) we have

$$
d t_{1} d \mu^{\prime}\left(u_{1}\right) \cdots d t_{p} d \mu^{\prime}\left(u_{p}\right)=d t d s_{1} \cdots d s_{p-1} d \mu^{\prime}\left(u_{1}\right) \cdots d \mu^{\prime}\left(u_{p}\right)=d t d \lambda_{p}(v)
$$

where

$$
d \lambda_{p}(v)=d s_{1} \cdots d s_{p-1} d \mu^{\prime}\left(u_{1}\right) \cdots d \mu^{\prime}\left(u_{p}\right) .
$$

Since $t$ is symmetric in $\left(t_{1}, \cdots, t_{p}\right)$, the transformation $(\epsilon)$ will leave $t$ invariant, and we have 


$$
(\epsilon)(t, v)=(t,(\bar{\epsilon}) v),
$$

where $(\overline{\boldsymbol{\epsilon}})$ is defined as follows. Given $t$ and $v=\left(s_{1}, \cdots, s_{p-1}, u_{1}, \cdots, u_{p}\right)$, let $\left(t_{1}, t_{2}, \cdots, t_{p}\right)$ be the solution of (5.5) and (5.6) and set

$$
(\bar{\epsilon}) v=\left(s_{1}^{\prime}, \cdots, s_{p-1}^{\prime}, u_{1}, \cdots, u_{p}\right), \quad s_{i}^{\prime}=\sum_{j} \alpha_{i j} t_{\epsilon(j)}, \quad i=1, \cdots, p-1 .
$$

It follows from (5.7) and (5.8) that $(\bar{\epsilon}) v$ is quite independent of $t$, so that we may consider $(\overline{\boldsymbol{\epsilon}}) \boldsymbol{v}$ to be a transformation on $\boldsymbol{v}$.

Since the transformation $(\tau)$ will leave $s_{i}$ and accordingly $v$ invariant by (5.7), we have

$$
(\tau)(t, v)=(t+\tau, v) .
$$

As the transformation $(\epsilon)$ preserves the measure $d t_{1} \cdots d t_{p} d \mu^{\prime}\left(u_{1}\right) \cdots$ $d \mu^{\prime}\left(u_{p}\right)$, we have

$$
d t d \lambda_{p}((\bar{\epsilon}) v)=d t d \lambda_{p}(v) \text { i.e. } d \lambda_{p}((\bar{\epsilon}) v)=d \lambda_{p}(v) .
$$

Hereafter we shall consider the functions in $L_{p}^{2}$ with respect to these new coordinates $(t, v)$. Then we see that the condition

$$
f(t,(\bar{\epsilon}) \boldsymbol{v})=f(t, v)
$$

is necessary and sufficient for $f$ to belong to $\tilde{L}_{p}^{2}$.

We consider the $L^{2}$-space over the measure space $\left(R^{2 p-1}, d \lambda_{p}\right)$ which will be denoted by $\mathcal{L}_{2 p-1}^{2}$. The totality of the functions in $\mathcal{L}_{2 p-1}^{2}$ invariant under the group $\{(\bar{\epsilon})\}$ constitutes a closed linear manifold in $\mathcal{L}_{2 p-1}^{2}$, say $\tilde{\mathcal{L}}_{2 p-1}^{2}$. Excluding the trivial case that $X(t, \omega)=\gamma t+\alpha$, the measure $d \lambda_{p}$ is not identically zero for $p=1,2, \cdots$, so that $\tilde{\mathcal{L}}_{2 p-1}^{2}$ is at least one-dimensional. In addition to this, the dimension of $\tilde{\mathcal{L}}_{2 p-1}^{2}$ is at most countable. Let $\tilde{\phi}_{p i}(v), i=1,2, \cdots$, be a complete orthonormal system in $\tilde{\mathcal{L}}_{2 p-1}^{2}$. Then $f(t, v) \in \tilde{L}_{p}^{2}$ is expressed as a sum of orthogonal components:

$$
f(t, v)=\sum_{n} f_{n}(t) \tilde{\phi}_{p n}(v),
$$

and we have

$$
\begin{aligned}
\|f(t, v)\|^{2} & =\sum_{n}\left\|f_{n}\right\|^{2} \\
\left(\tilde{S}_{\tau}^{(p)} f\right)(t, v) & =f(t+\tau, v)=\sum_{i} f_{i}(t+\tau) \tilde{\phi}_{p i}(v) .
\end{aligned}
$$

Thus $\tilde{L}_{p}^{2}$ is the direct sum of at most a countable number $(>0)$ of subspaces which reduce $\left\{\widetilde{S}_{\tau}^{(p)}\right\}$ and on each of which $\tilde{S}_{\tau}^{(p)}$ acts just as the unitary transformation $f(t) \rightarrow f(t+\tau)$ does on $L^{2}\left(R^{1}\right)$.

Summing up the above arguments we obtain the following

FUNDAMENTAL THEOREM. Except for the trivial case $X(t, \omega)=\gamma t+\alpha, H_{X}$ is isomorphic to the direct sum of the complex number field $C$ (one-dimensional 
Hilbert space) and a countable number of Hilbert spaces, each isomorphic to $L^{2}\left(R^{1}\right)$, i.e.

$$
H_{X} \cong C \oplus L^{2}\left(R^{1}\right) \oplus L^{2}\left(R^{1}\right)+\cdots,
$$

in such a way that this isomorphism transforms $U_{\tau}$ into the following operator:

$$
\Phi_{\tau}:\left(c, f_{1}(t), f_{2}(t), \cdots\right) \rightarrow\left(c, f_{1}(t+\tau), f_{2}(t+\tau), \cdots\right) .
$$

In other words, $\left\{U_{\tau}\right\}$ has spectra [2] of multiplicity one over the unitary measure and of uniform multiplicity $\boldsymbol{\aleph}_{0}$ over the ordinary Lebesgue measure and only these spectra.

\section{REFERENCES}

1. J. L. Doob, Stochastic processes, New York, 1952.

2. P. R. Halmos, Introduction to Hilbert space and the theory of spectral multiplicity, New York, 1951.

3. K. Itô, On stochastic processes, Jap. J. Math. vol. 18 (1942) pp. 261-301. 169.

4. - Multiple Wiener integral, Jour. of Math., Soc. of Japan. vol. 3 (1951) pp. 157-

5. — Complex multiple Wiener integral, Jap. J. of Math. vol. 22 (1952) pp. 63-86.

6. S. Kakutani, Determination of the spectrum of the flow of Brownian motion, Proc. Nat. Acad. Sci. U.S.A. vol. 36 (1950) pp. 319-323.

7. P. Lévy, Theorie de l'addition des variables aléatoires, Paris, 1937, rev. ed., 1954.

8. J. v. Neumann, Zur Operatorenmethode der klassischen Mechanik, Ann. of Math. vol. 33 (1932) pp. 587-648.

P. R. Halmos and J. v. Neumann, Operator methods in classical mechanics, II, Ann. of Math. vol. 43 (1942) pp. 332-350.

9. I. E. Segal, Tensor algebras over Hilbert spaces. I, Trans. Amer. Math. Soc. vol. 81 (1956) pp. 106-134.

Princeton University,

Princeton, N. J. 